\title{
Virus del papiloma humano, sus implicaciones en cavidad bucal: una revisión de la literatura
}

\author{
Human papillomavirus, its implications \\ in the oral cavity: Literature review
}

\author{
María Verónica Cuevas-González ${ }^{1}$, María Elisa Vega-Memije², \\ Graciela Zambrano-Galván ${ }^{3}$, Alma Graciela García-Calderón ${ }^{4}$, \\ Lilia Haidé Escalante-Macías ${ }^{3}$, Francisco Germán Villanueva-Sánchez ${ }^{3}$, \\ Ixchel Araceli Maya-García ${ }^{5}$, Juan Carlos Cuevas-González ${ }^{4} \bowtie$
}

\footnotetext{
${ }^{1}$ Universidad Nacional Autónoma de México, Ciudad de México.

${ }^{2}$ Departamento de Dermatología, Hospital General Dr. Manuel Gea González, Ciudad de México.

${ }^{3}$ Facultad de Odontología, Universidad Juárez del Estado de Durango, Durango, México.

${ }^{4}$ Departamento de Estomatología, Instituto de Ciencias Biomédicas, Universidad Autónoma de Ciudad Juárez, Ciudad Juárez, Chihuahua, México.

${ }^{5}$ Facultad de Odontología, Universidad Autónoma de Campeche, Campeche, México.
}

Departamento de Estomatología, Instituto de Ciencias Biomédicas, Universidad Autónoma de Ciudad Juárez, Anillo Envolvente del Pronaf s/n, Zona Pronaf, 32315 Cd. Juárez, Chihuahua, México. Teléfono: Tel. (+52) 688-2100 al 09. Correo electrónico: juan.cuevas@uacj.mx

Recibido: 8 de septiembre del 2017 Aprobado: 15 de noviembre del 2017 Disponible en línea: 1 de enero del 2018

Cómo citar este artículo: Cuevas González MV, Vega Memije ME, Zambrano Galván G, García Calderón AG, Escalante Macías LH, Villanueva Sánchez FG, et al. Virus del papiloma humano, sus implicaciones en cavidad bucal: una revisión de la literatura. Rev Nac Odontol. 2018;14(27), enero 2018. doi: https://doi.org/10.16925/od.v14i27.2346

\section{Resumen}

Introducción: divulgar las principales características clínicas de la afectación de algunos virus del papiloma humano en cavidad bucal permite al profesional de la salud identificar factores de riesgo, así como las principales lesiones asociadas con este virus. Esto con el fin de brindar atención oportuna y evitar así pasar por alto este tipo de lesiones.

Características: el virus del papiloma humano (vpH) pertenece a la familia de los Papillomaviridae, mide entre 50 y 55 nm de diámetro, son virus pequeños de ADN no envueltos, epiteliotrópicos cuyo genoma está formado por cadenas dobles de CADN. Este virus se puede llegar a encontrar de forma latente en el organismo durante años sin evidenciar cambios histológicos o clínicos, o bien puede formar papilomas benignos o verrugas cuyas lesiones consisten en tejido hiperplásico con engrosamiento de la capa espinosa del epitelio y un aumento de la proliferación capilar.

Resultados: al tratarse de un virus exclusivamente epiteliotrópico, la vía de entrada ocurre a través de microrrupturas del epitelio que exponen a las células de la superficie basal al virus, lo cual explica la alta frecuencia con la que se presenta en las poblaciones.

Conclusiones: el odontólogo de práctica general debe conocer las generalidades de los vPH, de tal manera que cuando identifiquen alguna lesión relacionada con algunos de los subtipos esté en capacidad de orientar al paciente y ofrecer un tratamiento acertado cuando así se requiera.

Palabras clave: características clínicas, cavidad bucal, papiloma, vPH. 


\title{
Human papillomavirus, its implications in the oral cavity: Literature review
}

\begin{abstract}
Introduction: Disclosing the main clinical characteristics of some human papilloma viruses implications in the oral cavity allows health professionals to identify risk factors, as well as the main lesions associated with the virus, with the objective of providing timely treatment and thus avoid overlooking this type of injury.

Characteristics: Human papillomavirus (HPV) belongs to the family of Papillomaviridae, it measures between 50 and $55 \mathrm{~nm}$ in diameter. It consists of small, untwisted, epitheliotropic DNA viruses; its genome consists of double-stranded CDNA. This virus can remain latent in the body for years without showing histological or clinical changes. It may also form benign papillomas or warts, these lesions consist of hyperplastic tissue, thickening of the epithelium's spinous layer, and an increase in capillary proliferation.
\end{abstract}

Results: Since the virus is exclusively epitheliotropic, its contagion occurs through microrruptures of the epithelium that expose basal surface cells to the virus, which explains the high frequency with which it presents in populations.

Conclusions: General dentists should know the basic facts of HPV, so that when they identify any injury related to some of the HPV subtypes, they may guide the patient and offer an appropriate treatment when required.

Keywords: clinical characteristics, oral cavity, papilloma, HPV.

\section{Vírus do papiloma humano, suas implicações na cavidade oral: uma revisão da literatura}

\section{Resumo}

Introdução: a divulgação das principais características clínicas das consequências de alguns vírus do papiloma humano na cavidade oral, permite ao profissional de saúde identificar fatores de risco, assim como as principais lesões associadas ao vírus. Isso, a fim de fornecer atenção oportuna e, assim, evitar ignorar esse tipo de lesão.

Características: 0 vírus do papiloma humano (VPH) pertence à família dos Papillomaviridae e mede entre 50 e 55 nm de diâmetro. Estes vírus são pequenos, de tipo ADN não envolto, epiteliotrópicos e 0 seu genoma é formado por cadeias duplas de CADN. Este vírus pode se encontrar de forma latente no corpo durante anos sem mostrar alterações histológicas ou clínicas, ou pode formar papilomas benignos ou verrugas cujas lesões consistem em tecido hiperplásico com espessamento da camada espinhosa do epitélio, assim como aumentar a proliferação capilar.

Resultados: por ser um vírus exclusivamente epiteliotrópico, a via de entrada é através de microrroturas do epitélio que deixam expostas as células da superfície basal ao vírus. Isso explica a alta frequência com que este vírus se apresenta nas populações.

Conclusões: o dentista clínico geral deve conhecer as generalidades do vPH de forma que, ao identificar uma lesão relacionada a alguns dos subtipos, seja capaz de orientar o paciente e oferecer um tratamento adequado quando necessário.

Palavras-chave: características clínicas, cavidade oral, papiloma, vPH. 


\section{Introducción}

El virus del papiloma humano (VPH) pertenece a la familia de los Papillomaviridae, mide entre 50 y $55 \mathrm{~nm}$ de diámetro, son virus pequeños de ADN no envueltos, epiteliotrópicos cuyo genoma está formado por cadenas dobles de CADN [1-3]. Este virus se puede llegar a encontrar de forma latente en el organismo durante años sin evidenciar cambios histológicos o clínicos, o bien puede llegar a formar lesiones papilomatosas benignas o verrugas cuyas lesiones consisten en tejido hiperplásico con engrosamiento de la capa espinosa del epitelio y un aumento de la proliferación capilar [4]. Al tratarse de un virus exclusivamente epiteliotrópico, la vía de entrada ocurre a través de microrrupturas del epitelio que exponen a las células de la superficie basal al virus. Si bien el receptor que permite la entrada aún es desconocido, el sulfato de heparina actúa como mediador de unión entre el virus y la célula $[5,6]$. Dicha infección deja entre 20 y 100 copias extracromosomales por célula. Una vez que el virus entra a la célula se forman dos proteínas: E6 y E7. La E6 se une a la ubiquitina celular, unión que resulta en la ubiquitinización de p53 con la subsecuente degradación proteolítica [4,7-9]. Se conocen al menos 200 subtipos de VPH, y se dividen en virus de bajo y alto riesgo, tal como se muestra en la tabla 1 [10-12].

El VPH es la infección de transmisión sexual más prevalente en el mundo, responsable de muchos tipos de cáncer como, por ejemplo, el cervico-uterino, el anal y el de pene. Este virus ha sido identificado en la zona de la cabeza y el cuello, con mayor frecuencia en orofaringe, y son los subtipos 16 y 18 los más relacionados en mayor grado en un $86,4 \%$ [13]. En el 2004 se describió que en el cáncer de laringe se ha identificado en un rango que va del 2,7\% al 46,9\% [14], y en el carcinoma de células escamosas de un $40 \%$ hasta un $80 \%[15,16]$. La epidemiología que se ha reportado en México indica que la presencia de VPH en mujeres va del $14,4 \%$ al $43 \%$ [17]; sin embargo, son escasos los datos que se conocen acerca la presencia de este virus en la cavidad bucal.

Los carcinomas de bucales ocupan el sexto lugar de todas las neoplasias malignas, y la incidencia anual se estima en 275,000 casos [18]. Los factores de riesgo para el desarrollo de este tipo de neoplasias se clasifican en genéticos y epigenéticos. Si bien dentro de estos últimos el tabaco y el alcohol son los primordiales, la dieta, la radiación y la inmunosupresión, entre otros, juegan un papel importante $[19,20]$. En los últimos años se ha visto un aumento en la asociación del VPH con el cáncer bucal, y se adjudica dicha aparición a cambios en la conducta sexual en pacientes jóvenes. El tabaquismo incrementa de forma significativa el riesgo de contraer VPH en ambos sexos debido a que aumenta los niveles de inflamación, de manera que disminuyen los mecanismos de respuesta inmune de la mucosa bucal [21, 22]. Sin embargo, esta relación no está del todo determinada, ya que Farsi et al. mencionaron que los efectos del tabaquismo y el alcoholismo intensos son independientes a la presencia del VPH, por lo que esta asociación aún debe de esclarecerse [23].

\section{A. Características clínicas}

El VPH genera una serie de lesiones papilomatosas en piel, así como en las mucosas anal, genital y bucal [24]. En tegumentos este virus se puede llegar a encontrar en lesiones tales como verrugas, queratosis actínica, cáncer de piel no melanoma, y en piel sana [25]. Las diferentes alteraciones que se relacionan con la presencia de los diversos subtipos de VPH se especifican en la tabla 2 [4].

\section{B. Lesiones benignas}

Entre las lesiones benignas con las que se ha relacionado la presencia de VPH se encuentran las verrugas vulgares (figura 1), las cuales se presentan en los jóvenes y afectan, por lo general, el tejido epitelial y membranas mucosas $[25,26]$. Lo causan los subtipos 2, 4 y 40 [27]. Cuando se presenta en la mucosa bucal las lesiones se encuentran en el borde bermellón, la mucosa labial o lengua, y se caracteriza por ser una lesión papilar o verrucosa indolora de base pediculada o sésil de coloración blanquecina o rosácea (suelen ser solitarias o múltiples) [28 29].

El condiloma acuminado es una lesión que se presenta con mayor frecuencia después de la segunda década de vida. Afecta a menudo la región genital, rectal y mucosa uretral; cuando se presenta en cavidad bucal se aprecia en la zona gingival, en las mejillas, los labios y el paladar duro $[30,31]$. La principal vía de transmisión son las prácticas sexuales orogenitales contagiadas por el VPH; el subtipo 11 es el que está relacionado con 
el desarrollo de esta lesión [32]. Clínicamente se asemeja a una coliflor con una superficie digitiforme de base sésil y crecimiento lento, su coloración va del rosado al blanco dependiendo del grado de queratinización [33, 34]. La hiperplasia epitelial multifocal o enfermedad de Heck es una enfermedad poco común que afecta principalmente a niños $[35,36]$, se localiza en la mucosa bucal, labios o lengua y, clínicamente, se caracteriza por la presencia de pápulas múltiples que dan apariencia de "empedrado" de consistencia blanda y coloración similar a la mucosa adyacente, y es el VPH serotipo 13 el asociado (véase la figura 1A) [37, 38].

\section{Lesiones potencialmente cancerizables}

Las lesiones potencialmente cancerizables, como, por ejemplo, la leucoplasia, se definen como una lesión blanca de la mucosa oral que no puede ser caracterizada como ninguna otra alteración ni clínica, ni histopatológicamente [39, 40]. Esta lesión ha tenido una relación poco clara con la presencia del VPH. Feller et al., en el 2012, mencionaron que el vPH subtipo 16 se considera de alto riesgo, y es el que se ha relacionado con la presencia de la leucoplasia idiopática y la leucoplasia verrucosa proliferativa [41]. Sin embargo, Bhargava et al. al determinar la relación del VPH en lesiones leucoplásicas orales no encontraron la presencia de este virus [42], por lo que la existencia del VPH en la leucoplasia aún no se encuentra bien definida.

En cavidad bucal el VPH puede desarrollar tres tipos de infecciones virales: clínica, la cual consiste en cambios macroscópicos de la membrana mucosa acompañados por imágenes microscópicas características; infección subclínica, en la cual es evidente la ausencia de cambios macroscópicos; y, por último, la infección latente (la más común), la cual se identifica por la ausencia de signos clínicos-patológicos, de manera que la presencia de dicho virus no puede confirmarse mediante estudios histopatológicos o citológicos [43]. Debido a que la presencia de dicho virus no siempre se evidencia se han establecido diferentes métodos diagnósticos para la identificación certera y temprana del VPH. Razavi et al. determinaron la sensibilidad de la técnica de PCR para la identificación de VPH E6 al evaluar 50 carcinomas faríngeos, y obtuvieron como resultado que el 36\% de los tejidos fueron positivos para la presencia del virus; concluyeron que la técnica de la reacción de la polimerasa en cadena es de gran utilidad para la detección, la cuantificación y la genotipificación del VPH [44]. Tambien se ha planteado el uso de biofluidos (por ejemplo, la saliva) a fin de realizar diagnósticos tempranos. Wang et al. analizaron la presencia de VPH en plasma y saliva de pacientes con carcinoma de células escamosas de cabeza y cuello, y reportaron 30 lesiones tumorales positivas a VPH-16, de las cuales el $40 \%$ se logró identificar en saliva [45]. De la misma forma, Tang et al. identificaron citoqueratinas como biomarcadores de carcinomas de células escamosas de cabeza y cuello positivos para VPH, y reportaron que en aquelos pacientes con carcinoma de células escamosas con presencia de VPH se identificaron niveles bajos de presencia de citoqueratina 8 y 18; concluyen que la expresión aberrante de citoqueratinas en saliva puede servir como un biomarcador en la identificación de VPH bucal [46]. Por otra parte, Khyani et al. analizaron la presencia de VPH 16 y 18 en saliva en población pakistaní, y observaron una baja prevalencia del virus; esto lo atribuyeron, principalmente, a la cultura de la población estudiada [47].

La participación que tiene el odontólogo como primera línea de atención de la cavidad bucal es de gran relevancia para la identificación temprana de las lesiones mencionadas, por lo que actualizar el conocimiento acerca del VPH en la mucosa orofaríngea es una necesidad cada vez mayor. Rajiah et al. llevaron a cabo un estudio en el cual se evaluó el nivel de conocimiento de los estudiantes de odontología acerca de los factores de riesgo, los síntomas y los métodos de prevención del carcinoma relacionado con el VPH, y demostraron un nivel de conocimiento moderado [48]. Por otra parte, Clarke et al. realizaron una evaluación a higienistas dentales y odontólogos de Canadá en la que analizaron cuál es su conducta al momento de realizar valoraciones para la detección de cáncer bucal. Mencionan que el profesional de la salud no se siente cómodo al explicar a los pacientes cuáles son los riesgos de la presencia de VPH en la cavidad bucal, y esto se debe a la naturaleza sexual del contagio [49]. Con base en lo anterior, es cierto que aún en la actualidad existen temas tabú difíciles de abordar, sin embargo, concientizar a nuestros pacientes acerca de la forma de contagio del virus y los riesgos que trae consigo dicha presencia en la cavidad bucal propiciará una disminución importante en la incidencia de neoplasias malignas originadas por VPH. Poelman et al. mencionaron una serie de puntos acerca del papel del odontólogo 
ante dicho virus, en los cuales incluyen la importancia de informar a nuestros pacientes acerca de la presencia del VPH, discutir acerca de los pros y contras de la vacunación contra el virus, así como realizar diagnósticos tempranos de lesiones potencialmente sospechosas [50].

\section{Conclusiones}

La incidencia de cáncer bucal a consecuencia del VPH aumenta de forma rápida alrededor del mundo, de forma tal que se llega a catalogar como "epidemia" en Norte América. Sin duda, el odontólogo como especialista en el área bucal se convierte

Tabla 1. Distintos tipos de VPH clasificados según el potencial de riesgo

\begin{tabular}{lc}
\hline VPH de bajo riesgo & \multicolumn{1}{c}{ VPH de alto riesgo } \\
\hline $6,11,42,43$ y 44 & $16,18,31,33,35,39,45,51,52,56$, \\
& $58,59,68,73$ y 82. \\
\hline
\end{tabular}

Fuente: elaboración propia en el primer contacto en la identificación de alguna alteración que se presente en la cavidad bucal, por lo cual actualizar el conocimiento acerca de este tema es de gran importancia a fin de estar en capacidad de identificar de forma oportuna cualquier cambio en las mucosas, o bien a pacientes con alto riesgo de desarrollar lesiones a causa del VPH.

Tabla 2. Subtipos de VPH y su relación con lesiones clínicas y potencialmente cancerizables o malignas

\begin{tabular}{lc}
\hline \multicolumn{1}{c}{ Lesiones clínicas } & Sub tipo de VPH \\
\hline Lesiones benignas & \\
$\begin{array}{l}\text { Papiloma bucal de células escamosas } \\
\text { (figura 1B) }\end{array}$ & 6 y 11 \\
Verruga vulgar & $1,2,4,7$, y 57 \\
Condiloma acuminado & 2,6 y 11 \\
Hiperplasia epitelial focal & 13 y 32 \\
\hline Lesiones potencialmente cancerizables o malignas \\
Leucoplasia & 6,16 y 18 \\
Carcinoma bucal y bucofaríngeo & 16 y 18 \\
Papilomatosis respiratoria recurrente & 6 y 11 \\
\hline
\end{tabular}

Fuente: elaboración propia

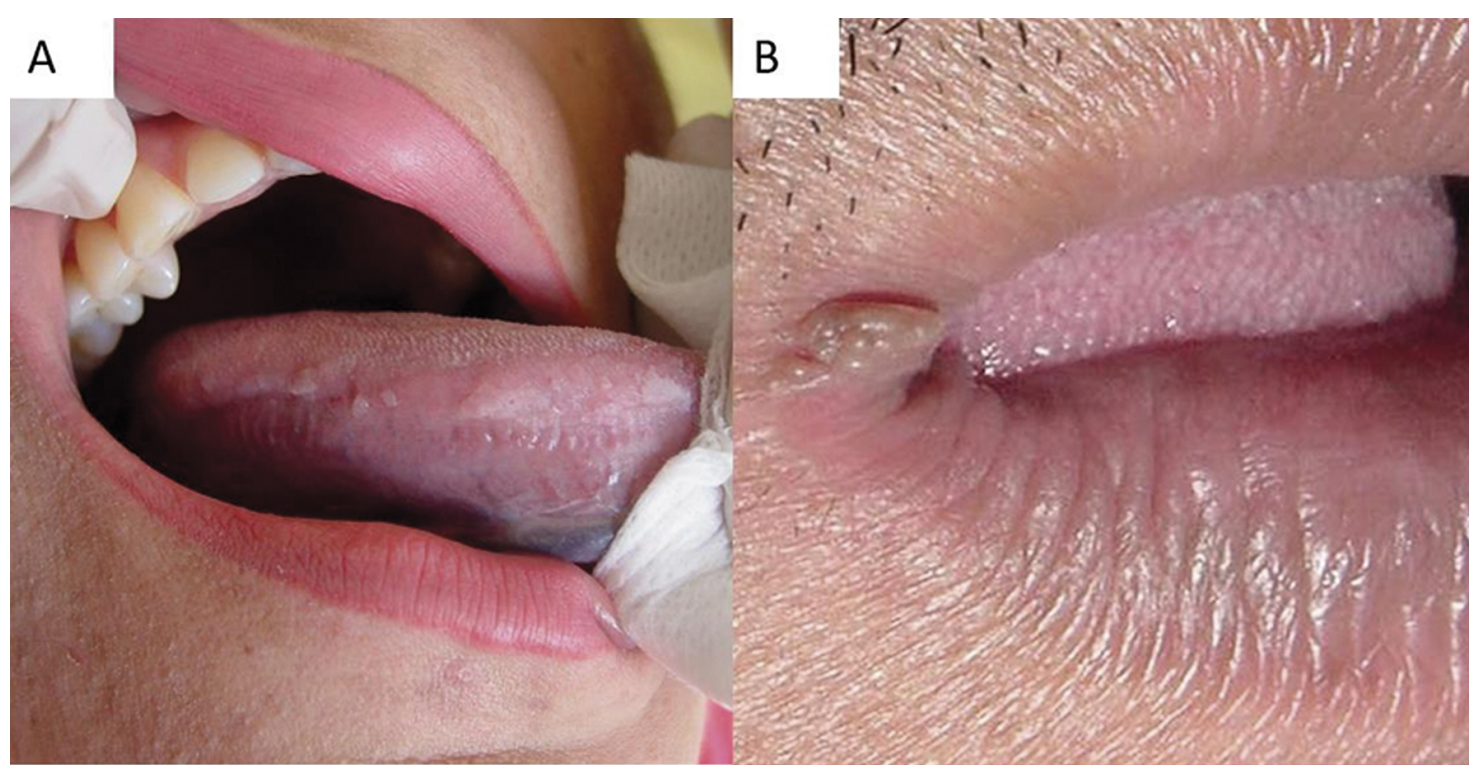

Figura 1. A. Múltiples verrugas vulgares localizadas en borde lateral de lengua. B. Verruga vulgar localizada en comisura labial previa a remoción quirúrgica

Fuente: elaboración propia 

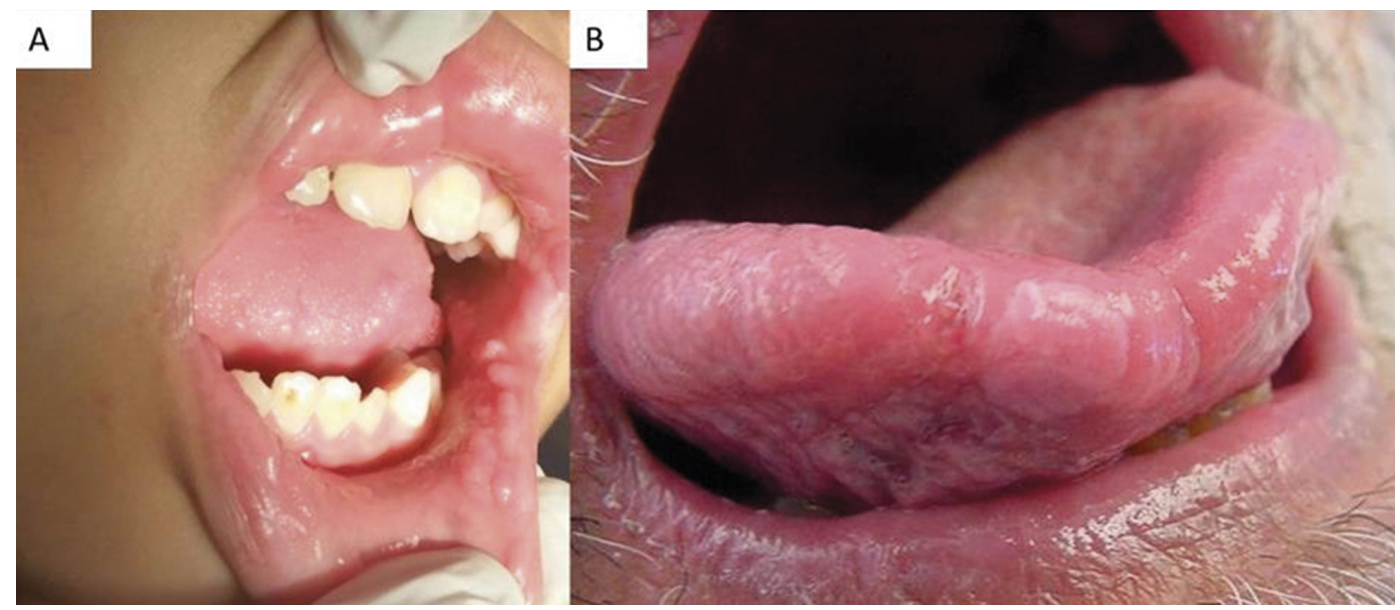

Figura 2. A. Lesiones múltiples de larga evolución asociadas al vPH tipo 13. B. Papiloma bucal en el que se encuentra implicado los subtipos 6 y 1

Fuente: elaboración propia

\section{Referencias}

[1] Bansal A, Singh MP, Rai B. Human papillomavirusassociated cancers: a growing global problem. Int J Appl Basic Med Res. 2016;6(2):84-9. doi: https://doi. org/10.4103/2229-516X.179027

[2] Martínez-Martínez A, Baldiris-Ávila R, Díaz-Caballero A. Papiloma bucal producido por vPH y su relación con carcinoma. Rev Clín Med Fam. 2012;5(2):144-145. doi: https://doi.org/10.4321/S16 99-695X2012000200012.

[3] De villiers EM, Fauquet C. Broker Tr, Bernard HU, zur Hausen H. Classification of papillomaviruses. Virol. 2004;324:17-27.

[4] Khot KP, Deshmane S, Choudhari S. Human papilloma virus in oral squamous cell carcinoma-the enigma unravelled. Chin J Dent Res. 2016;19(1):1723. doi: https://doi.org/10.3290/j.cjdr.a35693

[5] Longworth MS, Laimins LA. Pathogenesis of human papillomaviruses in differentiating epithelia. Microbiol Mol Biol Rev. 2004;68(2):362-72.

[6] Joyce JG, Tung JS, Przysiecki CT, Cook JC, Lehman ED, Sands JA, et al. The L1 major capsid protein of human papillomavirus type 11 recombinant virus-like particles interacts with heparin and cell-surface glycosaminoglycans on human keratinocytes. J. Biol. Chem. 1999;274:5810-5822

[7] Sritippho T, Chotjumlong P, Iamaroon A. Roles of human papillomaviruses and p16 in oral cancer. Asian Pac J Cancer Prev. 2015;16(15):6193-200.

[8] Scheffner, M, Werness BA, Huibregtse JM, Levine AJ, Howley PM. The E6 oncoprotein encoded by human papillomavirus types 16 and 18 promotes the degradation of p53. Cell. 1990;63:1129-1136.
[9] Werness, BA, Levine AJ y Howley PM. Association of human papillomavirus types 16 and 18 E6 proteins with p53. Science. 1990;248:76-79.

[10] Ahmed HG, Bensumaidea SH, Ashankyty IM. Frequency of human papilloma virus (HPV) subtypes 31, 33, 35, 39 and 45 among Yemeni women with cervical cancer. Infect Agent Cancer. 2015;10:29. doi: https://doi.org/10.1186/s13027-015-0026-9.eCollection 2015.

[11] Taberna M, Mena M, Pavón MA, Alemany L, Gillison ML, Mesía R. Human papillomavirus-related oropharyngeal cancer. Ann Oncol. 2017 oct 1;28 (10):2386-2398.doi:https://doi.org/10.1093/annonc/ mdx304.

[12] Qatouseh LA, Sabri I, Alkhatib I, Atwa E, Arafat T. Detection of high-risk human papillomavirus genotypes 16 and 18 in head and neck squamous. Cell Carcinomas in Jordan. Asian Pac J Cancer Prev. 2017;18(5):1337-1341. doi: https://doi.org/10. 22034/APJCP.2017.18.5.1337

[13] Rutkoski H, Fowler B, Mooney R, Pappas L, Dixon $\mathrm{BL}$, Pinzon LM et al. Pilot test of survey to assess dental and dental hygiene student human papillomavirus-related oropharyngeal cancer knowledge, perceptions, and clinical practices. J Cancer Educ. 2017. doi: https://doi.org/10.1007/s13187-017-1165-Z

[14] Atighechi S, Meybodian M, Dadgarnia MH, Baradaranfar $\mathrm{MH}$, Behniafard $\mathrm{N}$. Investigating the prevalence of human papilloma virus in squamous cell carcinoma of the larynx and its correlation with disease prognosis. Iran J Otorhinolaryngol. 2016;28(86):197-202. 
[15] Spence T, Bruce J, Yip KW, Liu FF. HPV Associated head and neck cancer. Cancers (Basel). 2016;8(8). doi: https://doi.org/10.3390/cancers8080075

[16] Parshad S, Nandi S, Marwah N, Mehta P, Tripathi M, Netrapal, et al. Human papillomavirus 16 and 18 in squamous cell carcinoma of oral cavity and sexual practices: a pilot study at a tertiary care hospital of North India. Natl J MaxillofacSurg. 2015;6(2):185-9. doi: https://doi.org/10.4103/0975-5950.183857

[17] Sam Soto S, de la Peña y Carranza AO, Plascencia JL. Human papillomavirus infection and adolescence. Ginecol Obstet Mex. 2011;79(4):214-24.

[18] Hernández-Guerrero JC, Jacinto-Alemán LF, Jiménez-Farfán MD, Macario-Hernández A, Hernández-Flores F, Alcántara-Vázquez A. Prevalence trends of oral squamouscell carcinoma. Mexico City's General Hospital experience. Med Oral Patol Oral Cir Bucal. 2013;18(2):306-11.

[19] Kumar M, Nanavati R, Modi TG, Dobariya C. Oral cancer: etiology and risk factors: a review. J Cancer Res Ther. 2016;12(2):458-63. doi: https://doi. org/10.4103/0973-1482.186696

[20] Mes SW, Te Beest D, Poli T, Rossi S, Scheckenbach K, van Wieringen WN2, y col. Prognostic modeling of oral cancer by gene profiles and clinicopathological co-variables. Oncotarget. 2017 Jul 26;8(35):5931259323. doi: https://doi.org/10.18632/oncotarget. 19576

[21] Kudo Y, Tada H, Fujiwara N, Tada Y, Tsunematsu T, Miyake $\mathrm{Y}$ et al. Oral environment and cancer. Genes Environ. 2016;38:13. doi: https://doi.org/10.1186/s4 1021-016-0042-z

[22] Shigeishi H, Sugiyama M. Risk Factors for oral human papillomavirus infection in healthy individuals: a systematic review and meta-analysis. J Clin Med Res. 2016;8(10):721-9. doi: https://doi. org/10.14740/jocmr2545w

[23] Farsi NJ, Rousseau MC, Schlecht N, Castonguay G, Allison P, Nguyen-Tan PF, et al. Aetiological heterogeneity of head and neck squamous cell carcinomas: the role of human papillomavirus infections, smoking, and alcohol. Carcinog.2017 sep 28. doi: https:// doi.org/10.1093/carcin/bgx106

[24] McCord C, Xu J, Xu W, Qiu X, Muhanna N, Irish J et al. Association of human papilloma virus with atypical and malignant oral papillary lesions. Oral Surg Oral Med Oral Pathol Oral Radiol. 2014;117(6):72232. doi: https://doi.org/10.1016/j.oooo.2014.02.003

[25] Bzhalava D, Guan P, Franceschi S, Dillner J, Clifford G. A systematic review of the prevalence of mucosal and cutaneous human papillomavirus types. Virol. 2013;445(1-2):224-31. doi: https://doi. org/10.1016/j.virol.2013.07.015
[26] Ural A, Arslan S, Ersoz Ş, Değer B. Verruca vulgaris of the tongue: a case report with a literature review. Bosn J Basic Med Sci. 2014;14(3):136-8. doi: https:// doi.org/10.17305/bjbms.2014.3.29

[27] Arteaga F, Velazco N, Quiñónez B, Corredor A. Verrugas vulgares bucales múltiples. Reporte de un caso. Acta Odontol Venez. 2008;46(1):92-93.

[28] Nagaraj M. Verruca vulgaris of the tongue. J Maxillofac Oral Surg. 2013;12(3):329-32 doi: https://doi. org/10.1007/s12663-010-0097-5

[29] Orth G, Jablonska S, Favre M, Croissant O, Obalek S, Jarzabek-Chorzelska M, Jibard N. Identification of papillomavirus in butchers' warts. J Invest Dermatol. 1981;76:97-102. doi: https://doi.org/10.1111/15231747.ep12525394

[30] Percinoto AC, Danelon M, Crivelini MM, Cunha RF, Percinoto C. Condyloma acuminata in the tongue and palate of a sexually abused child: a case report. BMC Res Notes. 2014;7:467. doi: https://doi. org/10.1186/1756-0500-7-467

[31] Ashiru JO, Ogunbanjo BO, Rotowa NA, Adeyemi-Doro FAB, Osoba AO. Intraoral condylomata acuminata: a case report. Br J Vener Dis. 1983;59: 325-326.

[32] Delgado-Quiñones EG, Sandoval-Magaña MA, Guzmán-Pantoja JE, Valdivia-López. Condiloma bucal en lengua: presentación de un caso clínico y revisión bibliográfica. Arch Med Fam. 2011;13(1): 74-76.

[33] Cháirez-Atienzo P, Vega-Memije ME, ZambranoGalván G, García-Calderón AG, Maya-García IA, Cuevas-González JC. Presencia del virus papiloma humano en la cavidad oral: revisión y actualización de la literatura. Int. J. Odontostomat. 2015;9(2): 233 238. doi: https://doi.org/10.4067/S0718-381X20150 00200009

[34] Luciano R, Oviedo J. M. Virus del papiloma humano y cáncer bucal. Acta Odontol Venez. 2013;51(1):1-3.

[35] Mansouri Z, Bakhtiari S, Noormohamadi R. Extensive focal epithelial hyperplasia: a case report. Iran J Pathol. 2015;10(4):300-5.

[36] Borborema-Santos CM, Castro MM, Santos PJB, Talhari S, Astolfi-Filho S. Oral focal epithelial hyperplasia: report of five cases. Braz Dent J. 2006;17(1):79

[37] De Castro LA, de Castro JG, da Cruz AD, Barbosa $\mathrm{BH}$, de Spindula-Filho JV, Costa MB. Focal epithelial hyperplasia (Heck's disease) in a 57-year-old brazilian patient: a case report and literature review. J Clin Med Res. 2016;8(4):346-50. doi: https://doi. org/10.14740/jocmr2466w

[38] Saghafi-Khadem S. Multifocal epithelial hyperplasia, a rare oral infection in Asia: Report of twelve cases in Iran. Med Oral Patol Oral Cir Bucal. 2010;15(4):591-5. 
[39] Escribano-Bermejo M, Bascones-Martínez A. Leucoplasia oral: conceptos actuales. Av. Odontoestomatol. 2009;25(2):83-97.

[40] Martínez Sahuquillo Márquez A, Gallardo Castillo I, Cobos Fuentes MJ, Caballero Aguilar J, Bullón Fernández P. La leucoplasia oral. Su implicación como lesión precancerosa. Av. Odontoestomatol. 2008;24(1):33-44.

[41] Feller L, Lemmer J. Oral leukoplakia as it relates to HPV infection: a review. Int J Dent. 2012;2012:540561. doi: https://doi.org/10.1155/2012/540561

[42] Bhargava A, Shakeel M, Srivastava AN, Raza TS, Rizvi S, Varshney P. Role of human papilloma virus in oral leukoplakia. Indian J Cancer. 2016;53(1):206-9. doi: https://doi.org/10.4103/0019-509X.180812

[43] Bogusiak K, Kobos J. The role of human papillomavirus infection in the head and neck region and methods for its detection. Pol J Pathol. 2014;65(1):1-14.

[44] Razavi Nikoo H, Ardebili A, Ravanshad M, Rezaei F, Teimoori A, Khanizadeh, et al. E6-Specific detection and typing of human papillomaviruses in oral cavity specimens from iranian patients. Iran Biomed J. 2017;21(6):411-6.

[45] Wang Y, Springer S, Mulvey CL, Silliman N, Schaefer J, Sausen M, y col. Detection of somatic mutations and HPV in the saliva and plasma of patients with head and neck squamous cell carcinomas. Sci Transl Med. 2015;24;7(293):293ra104. doi: https:// doi.org/10.1126/scitranslmed.aaa8507
[46] Tang KD, Kenny L, Perry C, Frazer I, Punyadeera C. The overexpression of salivary cytokeratins as potential diagnostic biomarkers in head and neck squamous cell carcinomas. Oncotarget. 2017; 31;8(42):72272-72280. doi: https://doi.org/10.18632 /oncotarget.19731

[47] Khyani IA, Qureshi MA, Mirza T, Farooq MU. Salivary detection of human Papilloma virus 16 \& 18 in pre-malignant and malignant lesions of oral cavity: Is it feasible in Pakistani context of socio-cultural taboos? Pak J Med Sci. 2015; 31(5):1104-9. doi: https:// doi.org/10.12669/pjms.315.7093

[48] Ajiah K, Maharajan MK, Fang Num KS, How Koh RC. Knowledge about human papillomavirus and cervical cancer: predictors of HPV vaccination among dental students. Asian Pac J Cancer Prev. 2010 jul 25;8(6):1573-1579.

[49] Clarke AK, Kobagi N, Yoon MN. Oral cancer screening practices of Canadian dental hygienists. Int J Dent Hyg. 2018;16(2):38-45. doi: https://doi. org/10.1111/idh.12295

[50] Poelman MR, Brand HS, Forouzanfar T, Daley EM, Jager DHJ. Prevention of HPV-related oral cancer by dentists: assessing the opinion of dutch dental students. J Cancer Educ. 2017 jul 24. doi: https://doi. org/10.1007/s13187-017-1257-9 